\title{
HERZLICH WILLKOMMEN BEIM 15. TAO-KONGRESS IN GRAZ!
}

\section{Sehr geehrte Frau Kollegin, sehr geehrter Herr Kollege!}

ich möchte Sie heute herzlich zu unserem 15.TAO-Kongress mit dem hochaktuellen Generalthema „Herz-Shen“ einladen, der vom 27.-29. September 2018 in Graz stattfinden wird. Wir haben dieses Thema gewählt, da es wie kein anderes den Puls der Zeit trifft und auf die Vielzahl an psychosomatischen Problemen unserer PatientInnen eingeht. Von unterschiedlichsten Seiten wird „das HerzShen" beleuchtet, spannende Überlegungen zur Diagnose und bewährteVorgangsweisen für die Therapie werden aufgezeigt und praxisnah vorgestellt.

Daneben wird das Thema „myofasciale Zusammenhänge" aus der Sicht der TCM präsentiert. Aus diesem Thema leitet sich ja nicht nur ein Verständnis für die Meridiantheorie $a b$, sondern es ergibt sich eine Vielzahl an Therapieoptionen, die allesamt perfekt mit der Akupunktur zu kombinieren sind.
Wir hoffen, damit das Interesse vieler KollegInnen getroffen zu haben und freuen uns bereits jetzt auf spannende TCM-Tage in unserer schönen Stadt.

Ihnen allen wünsche ich erholsame und Qi-aufbauende Sommertage und sende Ihnen

herzliche kollegiale Grüße

Ihr

Prof. Dr. med. Leopold Dorfer

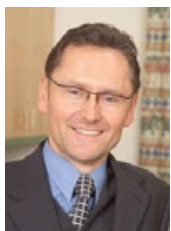

Prof. Dr. med. Leopold Dorfer

Präsident der Österreichischen Gesellschaft für Kontrollierte Akupunktur und TCM (OGKA)

Glacisstraße 7, A-8010 Graz

Tel. $+43316 / 374050$

E-Mail: office@ogka.at, Internet:www.ogka.at 